\title{
How auditory temporal processing deficits relate to dyslexia
}

\author{
C.F.B. Murphy and E. Schochat \\ Departamento de Fisioterapia, Fonoaudiologia e Terapia Ocupacional, Faculdade de Medicina, \\ Universidade de São Paulo, São Paulo, SP, Brasil \\ Correspondence to: C.F.B. Murphy, Av. Padre Antônio José dos Santos, 313, Apto. 134, 04563-010 São \\ Paulo, SP, Brasil \\ E-mail: crist78@yahoo.com
}

\begin{abstract}
Studies have shown that dyslexic children present a deficiency in the temporal processing of auditory stimuli applied in rapid succession. However, discussion continues concerning the way this deficiency can be influenced by temporal variables of auditory processing tests. Therefore, the purpose of the present study was to analyze by auditory temporal processing tests the effect of temporal variables such as interstimulus intervals, stimulus duration and type of task on dyslexic children compared to a control group. Of the 60 children evaluated, 33 were dyslexic (mean age $=10.5$ years) and 27 were normal controls (mean age $=10.8$ years). Auditory processing tests assess the abilities of discrimination and ordering of stimuli in relation to their duration and frequency. Results showed a significant difference in the average accuracy of control and dyslexic groups considering each variable (interstimulus intervals: $47.9 \pm 5.5$ vs $37.18 \pm 6.0$; stimulus duration: $61.4 \pm 7.6$ vs $50.9 \pm 9.0$; type of task: $59.9 \pm 7.9$ vs $46.5 \pm 9.0$ ) and the dyslexic group demonstrated significantly lower performance in all situations. Moreover, there was an interactive effect between the group and the duration of stimulus variables for the frequency-pattern tests, with the dyslexic group demonstrating significantly lower results for short durations $(53.4 \pm 8.2$ vs $48.4 \pm 11.1)$, as opposed to no difference in performance for the control group $(62.2 \pm 7.1 \mathrm{vs} 60.6 \pm 7.9)$. These results support the hypothesis that associates dyslexia with auditory temporal processing, identifying the stimulus-duration variable as the only one that unequally influenced the performance of the two groups.
\end{abstract}

Key words: Auditory temporal processing; Dyslexia; Hearing tests; Children

Research supported by FAPESP (\#02/09863-7).

Received August 23, 2008. Accepted May 4, 2009

\section{Introduction}

Several theories have been developed in order to discover the etiology of dyslexia, a reading problem. One of the hypotheses under study is based on auditory temporal processing deficiency. More specifically, this auditory temporal processing difficulty can be described as the limited ability to process "acoustic elements of short duration" - such as consonants with rapid formant transitions. Thus, dyslexic individuals appear to have difficulties in perceiving and distinguishing these sounds properly within the speech spectrum. Consequently, they would be unable to associate letters with their specific sounds (1-5).

Despite the large number of studies associating dyslexia with auditory temporal processing $(1,5-7)$, there still is some controversy about this association (8-11). According to Tallal (12), one of the pioneers of this hypothesis, one of the reasons for the existence of such doubts is related to the characteristics of the stimuli and to the tasks considered for the tests used.

For example, in the auditory processing tests employed (Repetition Test) in a study conducted by Tallal (1), children with dyslexia displayed the same level of performance as control children when the interstimulus intervals (ISIs) were long (428 ms). However, when the same stimuli were applied at reduced intervals ( 305 to $8 \mathrm{~ms}$ ), children with dyslexia presented a higher number of incorrect answers, not only for the sequencing test, but also for the discrimination test. This result was confirmed by the findings of Heiervang et al. (9), who also observed lower 
performance in the dyslexic group compared to the control group for short ISIs (8 to $150 \mathrm{~ms}$ ).

However, these results have been questioned by other researchers. Share et al. (10), for example, used the same tests applied by Tallal (1) on a group of 543 children who were evaluated while in the first grade and in later years. When compared to children without problems, children who presented reading difficulties later on displayed lower performance in all of the temporal processing tests conducted. Differences between groups were greater for long ISIs (428 ms), in contrast to the results published by Tallal (1). McAnally et al. (13) also investigated the temporal discrimination ability in tasks involving the frequency variable in good and poor readers. There were no differences between groups in interstimulus interval thresholds needed for discrimination.

Other temporal variables such as stimulus duration and task complexity are also being investigated. In a study conducted by Heiervang et al. (9), in which auditory processing tests were performed on a group of dyslexic children and on a control group, only the dyslexic children presented a significant drop in performance in cases of decreased stimulus duration and of comparison/ordering tasks with stimulus discrimination. Banai and Ahissar (14) obtained similar findings among dyslexic and control groups for tests involving simple stimulus discrimination and decreased performance (only the dyslexic group) in tasks involving identification or stimulus ordering.

The studies cited above are not in agreement concerning the way temporal parameters can influence dyslexic children in auditory processing tests. Thus, the objective of the present study was to analyze the effect of temporal variables such as ISI, stimulus duration and type of task on dyslexic children compared to a control group in auditory temporal processing tests. Our working hypothesis was that the parameters under study influence the two groups in a different manner, thus corroborating findings that relate dyslexia to an auditory temporal processing deficiency.

\section{Subjects and Methods}

\section{Subjects}

Data collection was conducted at the Auditory Processing Laboratory of the Speech Pathology and Audiology Course, University of São Paulo Medical School, from January 2003 to May 2004. The control group consisted of 27 children ( 15 girls and 12 boys; mean age $=10$ years and 8 months; range $=9$ years and 2 months to 12 years and 4 months), and the dyslexic group consisted of 33 children (14 girls and 19 boys; mean age $=10$ years and 5 months; range $=9$ years and 5 months to 12 years and 5 months). All subjects were raised in a monolingual Brazilian-Portuguese environment, studying at private schools in São Paulo city, which guaranteed a similar socioeconomic and cultural cross-section. All children received normal ratings in their basic audiometric evaluation (audiometry, speech audiometry and imitanciometry). Moreover, they did not demonstrate any cognitive, psychological, neurological, or ophthalmological disorders, or any oral language-acquisition retardation. Furthermore, they had no history of otitis and no musical knowledge.

The Brazilian Dyslexic Association was responsible for the diagnosis and referral of the group under study. The diagnosis was based on the following criteria: average or above-average intelligence according to the Wechsler Intelligence Scale for Children-III (WISC-III; 90 or more in verbal and non-verbal intelligence tests); reading and phonological-perception abilities 2 years behind those of ordinary children at that age; questionnaires were applied to identify any problems that could interfere with reading skills, such as educational or teaching methods. The control group consisted of children selected from private schools by teachers who were instructed to choose children without school problems. Since all the children chosen demonstrated good academic performance, they were presumed to have IQs of 90 or above on the WISC-III scale.

The study was approved by the Research Project Ethics Committee (CAPPesq) of the Hospital das Clínicas da Faculdade de Medicina da Universidade de São Paulo (case No. 649/01) on October 9, 2002, and the persons responsible for the children gave written informed consent to participate.

\section{Procedures}

Participants from both groups underwent a series of examinations: clinical history, otoscopy, imitanciometry, and speech and tonal audiometry. After being submitted to these tests, children that did not satisfy the criteria were excluded from the study and, when necessary, were referred to a specialized professional. After selecting the group, auditory temporal processing tests were conducted.

The auditory temporal processing tests were created at the Radio Laboratory of the University of São Paulo School of Communications and Arts (ECA). The tests were recorded with a MACKIE SR 32-4 mixer (USA) and an HP $8100 \mathrm{CD}$ recorder (USA), using the Sound Forge 4.5 software (USA) and recording directly onto the computer's hard disk using a Pentium processor. A female announcer introduced (identified) the tests using an SM81-LC (USA) microphone. The tests were conducted in an acoustically 
isolated booth, with a CD playing on the Windows Media Player of an LG computer (USA) with Pioneer SE-A20 (USA) speakers.

The tests were adapted from tests developed by Tallal and Piercy (15), known as "Repetition Test". The "Repetition Test" is composed of a series of tests in which several of the main components of temporal processing are examined separately, i.e., association and discrimination, ordering and perception speed. All tests present non-verbal (pure tone) stimuli only, which are given in pairs that differ in frequency $(\mathrm{Hz})$ alone. The duration is constant $(75 \mathrm{~ms})$. During the first phase (association and discrimination), the participant is instructed to separately associate each stimulus heard with its corresponding button on a response box. Thus, after having obtained a certain percentage of correct answers, the participant is presumed to be able to distinguish between the two stimuli and to associate them with a specific motor response, which shows that the participant is ready for the second phase (ordering). In the ordering phase, the participant is instructed to arrange in order a pair of perceived stimuli by associating each one with its corresponding button, in the same order in which they appeared. The interval between stimuli is constant (428 $\mathrm{ms})$. In the last phase (perception speed), as in the previous phase, the participant is instructed to order a pair of perceived stimuli. The difference is in the intervals between the stimuli, which vary randomly between 8 and 305 $\mathrm{ms}(8,15,30,60,150$, and $305 \mathrm{~ms})$.

As in the original test (15), this study's series of tests also included tests involving the discrimination and ordering of non-verbal (pure tone) stimuli of different frequency. Modifications were made in the following items:

Parameters present in each test. Stimulus duration and frequency amounts were selected from a pilot study of Brazilian children, in which the average accuracy in all tests was over $70 \%$ for children without language and/or hearing problems. The ISIs were chosen on the basis of the results obtained by Tallal and Piercy (15), which revealed performance differences between the test group and the control group for intervals between 50 and $428 \mathrm{~ms}$. Thus, in the present study, ISI durations were selected within this range.

Inclusion of tests involving the discrimination and ordering of stimuli of different durations ( $m s$ ). This procedure was adopted in order to observe the influence of the stimulus duration parameter on the performance of both groups under study.

Type of response. Contrary to the original test, in which auditory stimuli were associated with a motor response, in the present study the stimuli were associated with a verbal response, such as saying "equal" or "different" in the dis- crimination tests; or "bass" or "treble", in the ordering tests. This procedure was adopted to facilitate data collection.

Thus, based on the characteristics of the original test, and on the modifications made to it, the characteristics of each of the eight auditory tests performed were as follows:

Test 1: Frequency discrimination 1. The subject is asked to distinguish a pair of auditory stimuli with frequencies of 1000 or $1400 \mathrm{~Hz}$ (stimuli 1 and 2, respectively). As such, there are two possible verbal responses: "equal stimuli" (if the subject hears 1-1 or 2-2) or "different stimuli" (if the subject hears 1-2 or 2-1). The stimuli have a constant duration of $100 \mathrm{~ms}$ and ISIs that vary randomly between 50 and $250 \mathrm{~ms}(50,100,150,200$, and $250 \mathrm{~ms})$.

Test 2: Frequency discrimination 2. As in test 1, this test also examines the task of frequency discrimination. The only difference lies in the duration of the stimulus, which has a constant value of $200 \mathrm{~ms}$.

Test 3: Duration discrimination 1. Subjects are requested to distinguish a pair of stimuli that last 300 or 600 ms (stimuli 1 and 2, respectively). As such, there are two possible verbal responses: "equal stimuli" (if the subject hears 1-1 or 2-2) or "different stimuli" (if the subject hears 1-2 or 2-1). The stimuli have a constant frequency of 1000 $\mathrm{Hz}$ and ISls that vary randomly between 50 and $250 \mathrm{~ms}$ (50, 100, 150, 200, and $250 \mathrm{~ms})$.

Test 4: Duration discrimination 2. As in test 3, this test also examines the task of duration discrimination. The only difference lies in the duration of the stimuli (200 or $400 \mathrm{~ms}$ ).

Test 5: Frequency ordering 1. Subjects are requested to arrange in order a pair of stimuli that have a frequency of 1000 and $1400 \mathrm{~Hz}$ (stimuli 1 and 2, respectively). As such, there are four possible verbal responses: bass treble (if the subject hears 1-2), bass bass (if the subject hears 1-1), treble treble (if the subject hears 2-2), and treble bass (if the subject hears 2-1). The stimuli have a constant duration of $200 \mathrm{~ms}$ and ISIs that vary randomly between 50 and $250 \mathrm{~ms}(50,100,150,200$, and $250 \mathrm{~ms})$.

Test 6: Frequency ordering 2. As in test 5 , this test also examines the task of frequency ordering. The only difference lies in the duration of the stimulus, which has a constant value of $100 \mathrm{~ms}$.

Test 7: Duration ordering 1. Subjects are requested to arrange in order a pair of stimuli that have a duration of 300 or $600 \mathrm{~ms}$ (stimuli 1 and 2, respectively). As such, there are four possible verbal responses: long short (if the subject hears 2-1), short long (if the subject hears 1-2), short short (if the subject hears 1-1), and long long (if the subject hears 2-2). The stimuli have a constant frequency of $1000 \mathrm{~Hz}$ and ISIs that vary randomly between 50 and $250 \mathrm{~ms}(50,100$, 150, 200, and $250 \mathrm{~ms}$ ).

Test 8: Duration ordering 2. As in test 7, this test also 
examines the task of duration ordering. The only difference lies in the duration of the stimuli, which have values of 200 or $400 \mathrm{~ms}$, for stimulus 1 or 2 , respectively.

Based on Figure 1, it is possible to visualize the schematic representation of a test trial using the variables considered (duration, frequency and inter-stimulus interval). Figure 2 exemplifies four options of possible trials available in one of the tests (test 2 - frequency discrimination 2).

Each discrimination test consisted of 40 trials ( 40 pairs of stimuli) and each ordering test consisted of 30 trials (30 pairs of stimuli). Each stimulus displayed a rise and fall of $5 \mathrm{~ms}$.

While conducting the tests, the subject and researcher were next to each other in front of the computer. It was possible to see the display panel of the program used to play the $C D$ (track number, seconds remaining, and elapsed time) on the screen. Tests were performed at a comfortable intensity of approximately $70 \mathrm{dBHL}$, according to measurements performed using a sound pressure level meter. Stimuli were binaural, i.e., they were applied simultaneously to both ears. The researcher was responsible for registering the subjects' responses according to a specific protocol.

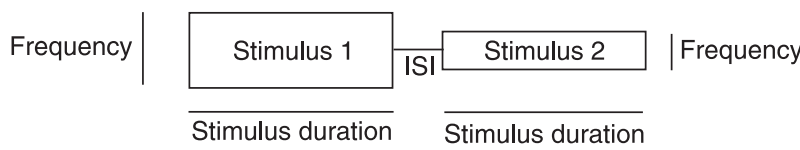

Figure 1. Schematic presentation of an example of a test with stimuli of the same duration and different frequencies ("treble bass"). Stimulus $1=1000 \mathrm{~Hz}$; stimulus $2=1400 \mathrm{~Hz}$. ISI = interstimulus interval.

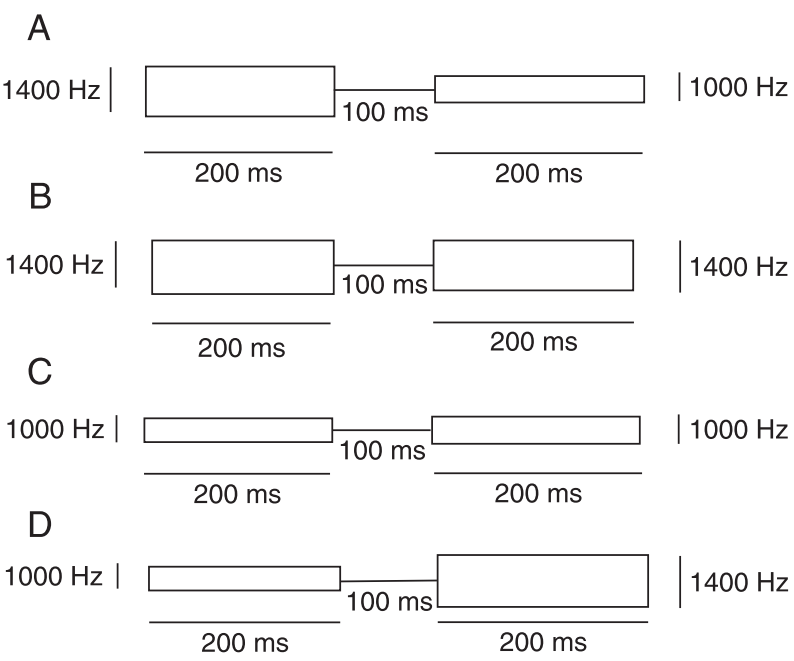

Figure 2. Example of the four options available in one of the tests (Test 2 - Frequency Discrimination 2): $A$, "bass treble"; $B$, "bass bass"; $C$, "treble treble"; $D$, "treble bass".

\section{Results}

Findings will be presented separately for each variable. For inferential analysis, analysis of variance with successive repetitions was adopted (16). When necessary, the Bonferroni method was used to continue the analysis. The level of significance was set at 0.05 .

\section{Interstimulus interval}

For the analysis of ISI, all tests performed presented pairs of stimuli separated by an interval varying randomly between 50, 100, 150, 200, and 250 ms. Thus, the number of correct answers in the eight tests was summed for ISI. Each ISI interval appeared 8 times in each discrimination test and 6 times in the ordering tests. Hence, a total of 56 events were counted for each interval.

According to the data shown in Table 1, there was a significant difference in mean accuracy between the two groups $(P=0.000)$, with the mean of the dyslexic group being lower than that of the control group. This was observed for all ISIs; that is, there was no significant interaction effect between group and ISI.

\section{Stimulus duration}

For the analysis of stimulus duration, the tests of the frequency pattern with 200-ms stimuli (tests 2 and 5) were compared to those with 100-ms stimuli (tests 1 and 6). Also, the tests with duration patterns of $300-\mathrm{ms}$ and 600 ms stimuli (tests 3 and 7) were compared to the 200-ms and 400-ms ones (tests 4 and 8).

Comparison between tests with stimuli of 100 and 200 ms: (test $1+6) \times($ test $2+5)$. According to the data presented in Table 2, the effect of duration on mean accuracy was not the same for the two groups $(P=0.021$; an effect of interaction between groups and duration was detected). Sequential analysis yielded the following results: in the control group, no significant difference in average accuracy was detected between the two durations; in the dyslexic group, mean accuracy was higher at $200 \mathrm{~ms}$ than at $100 \mathrm{~ms}(P=0.000)$; with the $100-\mathrm{ms}$ duration, the mean accuracy of the control group was higher than that of the dyslexic group $(P=0.000)$ and the same occurred with the 200-ms duration $(P=0.000)$.

Comparison between tests with stimuli of 300/600 and 200/400 ms: (test $3+7) x$ (test $4+8$ ). Table 3 shows significant differences in mean accuracy between the two durations $(P=0.000$ ), with the $300 / 600-m s$ mean being higher than the 200/400-ms mean. These differences did not depend on the group. The two groups differed in mean accuracy $(P=0.000)$, with the control group presenting a higher mean than the dyslexic group. 
Type of task (discrimination vs ordering)

In order to analyze the task-type variable, the stimulusdiscrimination tests (tests 1, 2, 3, and 4) were compared to the stimulus-ordering tests (tests $5,6,7$, and 8), considering their frequency and duration.
For this analysis, the amount of accuracy was a percentage, since each task involved a specific number of trials. The data described in Table 4 shows that the mean accuracy of the two tasks was not the same $(P=0.000)$, with the ordering task exhibiting a lower mean. The differ-

Table 1. Number of correct answers in the eight tests for both groups - accuracy as a function of interstimulus interval (ISI) and group.

\begin{tabular}{|c|c|c|c|c|c|}
\hline & $50 \mathrm{~ms}$ & $100 \mathrm{~ms}$ & $150 \mathrm{~ms}$ & $200 \mathrm{~ms}$ & $250 \mathrm{~ms}$ \\
\hline \multicolumn{6}{|l|}{ Control children $(N=27)$} \\
\hline Total number & 56 & 56 & 56 & 56 & 56 \\
\hline Mean & 47.2 & 47.4 & 48.5 & 48.6 & 48.0 \\
\hline Standard deviation & 5.3 & 5.0 & 5.0 & 5.9 & 5.5 \\
\hline Minimum & 33 & 37 & 38 & 35 & 35 \\
\hline Median & 48 & 48 & 49 & 51 & 49 \\
\hline Maximum & 56 & 55 & 56 & 56 & 56 \\
\hline \multicolumn{6}{|l|}{ Dyslexic children $(\mathrm{N}=33)$} \\
\hline Total number & 56 & 56 & 56 & 56 & 56 \\
\hline Mean & 36.1 & 37.1 & 37.7 & 37.8 & 37.2 \\
\hline Standard deviation & 6.0 & 6.2 & 6.4 & 5.8 & 6.4 \\
\hline Minimum & 23 & 27 & 25 & 26 & 23 \\
\hline Median & 36 & 37 & 38 & 38 & 38 \\
\hline Maximum & 51 & 55 & 50 & 51 & 52 \\
\hline Difference between groups ( $P$ value) & $<0.001^{*}$ & $<0.001^{*}$ & $<0.001^{*}$ & $<0.001^{*}$ & $<0.001^{*}$ \\
\hline
\end{tabular}

Total number $=$ total number of correct answers. Statistical test = analysis of variance with successive repetitions, level of significance $=0.05$.

Table 2. Number of correct answers in the eight tests for both groups - accuracy as a function of stimulus duration and group (frequency tests).

\begin{tabular}{lcc}
\hline & $100 \mathrm{~ms}$ & $200 \mathrm{~ms}$ \\
\hline $\begin{array}{l}\text { Control children }(\mathrm{N}=27) \\
\quad \text { Total number }\end{array}$ & 70 & 70 \\
Mean & 60.6 & 62.2 \\
Standard deviation & 7.9 & 7.1 \\
Minimum & 45 & 48 \\
Median & 63 & 64 \\
Maximum & 70 & 70 \\
Dyslexic children $(\mathrm{N}=33)$ & & \\
Total number & 70 & 70 \\
Mean & 48.4 & 53.4 \\
Standard deviation & 11.1 & 8.2 \\
Minimum & 27 & 33 \\
Median & 49 & 54 \\
Maximum & 70 & 70 \\
Difference between groups (P value) & $<0.001^{*}$ & $<0.001^{*}$ \\
Effect group and duration (P value) & $0.021^{*}$ & \\
\end{tabular}

Total number $=$ total number of correct answers. Statistical test $=$ analysis of variance with successive repetitions, level of significance $=0.05$
Table 3. Number of correct answers in the eight tests for both groups - accuracy as a function of stimulus duration and group (ordering tests).

$300 / 600 \mathrm{~ms} 200 / 400 \mathrm{~ms}$

$\begin{array}{lcc}\text { Control children }(\mathrm{N}=27) & & \\ \text { Total number } & 70 & 70 \\ \text { Mean } & 60.9 & 56.0 \\ \text { Standard deviation } & 7.6 & 8.8 \\ \text { Minimum } & 42 & 31 \\ \text { Median } & 63 & 56 \\ \text { Maximum } & 70 & 68 \\ \text { Dyslexic children }(\mathrm{N}=33) & & \\ \text { Total number } & 70 & 70 \\ \text { Mean } & 43.8 & 40.4 \\ \text { Standard deviation } & 8.7 & 7.1 \\ \text { Minimum } & 23 & 28 \\ \text { Median } & 44 & 39 \\ \text { Maximum } & 64 & 54 \\ \text { Difference between groups (P value) } & <0.001^{*} & <0.001^{*} \\ \text { Effect group and duration (P value) } & 0.431 & \end{array}$

Total number $=$ total number of correct answers. Statistical test $=$ analysis of variance with successive repetitions, level of significance $=0.05$ 
Table 4. Number of correct answers in the eight tests for both groups - accuracy as a function of order and group.

\begin{tabular}{lcc}
\hline & Discrimination & Ordering \\
\hline Control children $(\mathrm{N}=27)$ & 160 & 120 \\
$\quad$ Total number & 89.6 & 80.2 \\
Mean & 7.6 & 11.5 \\
Standard deviation & 71.9 & 55.0 \\
Minimum & 92.5 & 79.2 \\
Median & 100.0 & 99.2 \\
Maximum & & \\
Dyslexic children (N = 33) & 160 & 120 \\
Total number & 71.5 & 59.6 \\
Mean & 10.6 & 12.4 \\
Standard deviation & 53.1 & 40.0 \\
Minimum & 74.4 & 58.3 \\
Median & 90.6 & 95.0 \\
Maximum & $<0.001^{*}$ & $<0.001^{*}$ \\
Difference between groups (P value) & 0.288 & \\
Effect group and task (P value) & & \\
\hline
\end{tabular}

Total number $=$ total number of correct answers. Statistical test $=$ analysis of variance with successive repetitions, level of significance $=0.05$.

ence between the mean percentages of the two tasks was the same for both groups. The average percent accuracy was higher in the control group $(P=0.000)$.

\section{Discussion}

The objective of the present study was to determine the influence of temporal variables that are present in auditory processing tests applied to children with dyslexia.

First of all, the analysis showed that, independent of the variables present, there was a significant difference in performance between groups in all tests performed. In all cases, the average of the dyslexic group was lower than the average of the control group. Concerning the influence of the temporal variables, only the stimulus-duration variable (for the frequency-pattern tests) influenced the performance of both groups in a different way, since only the dyslexic group displayed performance that was significantly lower for the short-duration-stimulus tests than for the long-duration-stimulus tests (Table 2). As to the other variables analyzed, both groups exhibited significantly lower performance in tasks involving stimulus ordering than in stimulus-discrimination tasks (Table 3 ). The variation in ISIs did not influence the performance of either group (Table 1).

In all tests, the lower performance of the dyslexic group compared to the control group was expected considering the results obtained in other studies that also examined this relationship $(1,5-7,10)$. As was the case for the present study, all studies indicated the existence of an alteration related to auditory temporal processing in dyslexic children.

Concerning the influence of ISIs, a drop in the performance of the dyslexic group was expected between the intervals under study on the basis of results obtained in other studies $(1,9,17,18)$. In the investigation conducted by Tallal (1), which was one of the first studies in which similar tests were performed on the same cross-sectional group, the results showed that children with reading difficulties obtained the same amount of correct responses as the control group did for long-duration ISIs (428 ms). A difference was only observed with short ISIs (8 to $305 \mathrm{~ms}$ ). However, the investigator did not show whether the variation present between 8 and 305 ms influenced the performance of the dyslexic group, as was demonstrated in the present study. This analysis was conducted by Tallal and Piercy (15) on a group of children with specific language disorders. The results showed a significant drop only in the test group's performance for ISIs between 8 and $428 \mathrm{~ms}$, demonstrating that this interval exhibits an interactive effect between group and ISIs. Results similar to those of Tallal and Piercy (15) were obtained by Nagarajan et al. (18), whose control groups' responses were almost 100\% correct for all ISIs analyzed, while there was a drop in performance with decreasing ISIs for a group of dyslexic adults, with percentages varying from a correct-response rate of about $75 \%$ for ISIs of $500 \mathrm{~ms}$ to a rate of $60 \%$ for 100-ms ISIs. Heiervang et al. (9) also obtained similar results, with their dyslexic group achieving a lower performance than the control group for short ISIs (8 to $150 \mathrm{~ms}$ ).

The differences observed between the present results and those of the cited studies $(1,9,17,18)$ may be related to the characteristics of the individuals in the groups examined, such as their cognitive profiles and their native tongues.

Concerning the cognitive profile, in the present study all children in the dyslexic group took tests involving cognitive skills, and no scores below the expected average were observed. Nevertheless, the criteria employed in the selection of the children in the present study may not have been the same as those employed in the selection of the groups that participated in the other studies. Ben-Yehudah et al. (19), for example, studied the influence of cognitive skills in tests like those performed here and their results showed that this factor could influence the dyslexic group.

Concerning the influence of the native language of the participants, no other studies involving Portuguese-speaking children were identified in the literature. However, similar tests have been performed on native speakers of 
Hebrew (19) and German (20). Heim et al. (20) observed no influence on dyslexic or control groups for ISIs ranging from 8 to $305 \mathrm{~ms}$, although differences in performance were observed within each group. As mentioned previously, Ben-Yehudah et al. (19) also observed dyslexics with average cognitive skills who exhibited difficulties for all ISIs (100 ms to $2 \mathrm{~s}$ ).

Simon and Fourcin (21) showed that the linguistic environment interferes with the abilities of distinguishing and naming acoustic patterns. The authors discovered that English 4 year olds are capable of using the F1 transition path, whereas French children only use it at about nine years of age. The authors concluded, however, that this path is more important in the English language than in French. Therefore, although the present study and the studies cited above employed non-verbal stimuli, the way in which this stimulus is processed by speakers of different languages can vary (22).

The results obtained here concerning the influence of the duration of the stimuli were similar to those obtained by Tallal and Piercy (15). These investigators compared the performance of children with language acquisition disorder in two frequency-ordering tests, whereby each test applied three stimuli to be arranged in order, with durations of 75 and 250 ms. With 75-ms stimuli, all children in the control group exhibited performance up to the expected standard, while only $17 \%$ of the children in the dyslexic group displayed a performance similar to that of the control group. However, when 250-ms stimuli were applied, 83\% of the children in the dyslexic group demonstrated performance similar to that of the control group.

A similar analysis was performed by Heiervang et al. (9) using stimuli with 75 and $250 \mathrm{~ms}$ of duration in tests involving frequency discrimination and ordering. The results showed that both groups displayed lower performance with 75-ms stimuli, but this lower performance was more marked in the group of dyslexic children. The authors, however, discussed the importance of the duration of the stimuli as a variable to be considered in studies involving dyslexia, in agreement with the findings obtained in the present study.

Research into the influence of different tasks was also conducted by Tallal (1), who obtained results different from those of the present study. His results showed that both groups displayed lower performance in ordering tasks, but this was more marked for the dyslexic group. Banai and Ahissar (14) also compared the performance of a control group to that of a group of individuals with learning difficulties in tasks involving frequency. These investigators found no differences between the two groups in the performance of stimulus discrimination tasks. The only differences observed were in the performance of tasks involving ordering. The researchers concluded that the degree of difficulty observed in individuals with dyslexia could be determined by the type of task solicited. Heiervang (9) also obtained similar results. He performed modified Tallal-and-Piercy (15) tests on dyslexic and control group children, and noticed that only the test group displayed a much lower performance in discrimination tasks than in ordering tasks.

The difference between the present results and those obtained in the above-cited studies may be related to the great variability of responses obtained in the ordering-task tests of the current study, which can be demonstrated by the high variance pattern present in both groups. The absence of a pattern of specific responses for each group made it difficult to compare the groups. This type of performance was also observed by Berwanger et al. (23) and Frascá (24) in evaluations of control groups by testing and retesting.

The present study suggests that dyslexic children have difficulties with tasks involving auditory temporal processing and are influenced by the duration of the stimuli employed in the tests. Future studies are needed to investigate the relationship between the main symptoms observed in dyslexic children, such as phonological deficit, and the mechanisms responsible for deficits involving auditory temporal processing.

\section{References}

1. Tallal P. Auditory temporal perception, phonics, and reading disabilities in children. Brain Lang 1980; 9: 182-198.

2. Merzenich MM, Schreiner CS, Jenkins WM, Wang X. Temporal information processing in the nervous system: special reference to dyslexia and dysphasia. New York: New York Academy of Sciences; 1993.

3. Fitch RH, Miller S, Tallal P. Neurobiology of speech perception. Annu Rev Neurosci 1997; 20: 331-353.

4. Habib M. The neurological basis of developmental dyslexia: an overview and working hypothesis. Brain 2000; 123 (Part 12): 2373-2399.

5. Van Ingelghem $M$, van Wieringen A, Wouters J, Vandenbussche E, Onghena P, Ghesquiere P. Psychophysical evidence for a general temporal processing deficit in children with dyslexia. Neuroreport 2001; 12: 3603-3607.

6. Birch HG, Belmont L. Auditory-visual integration in normal 
and retarded readers. Am J Orthopsychiatry 1964; 34: 852861.

7. Talcott JB, Witton C, McLean MF, Hansen PC, Rees A, Green GG, et al. Dynamic sensory sensitivity and children's word decoding skills. Proc Natl Acad Sci U S A 2000; 97: 2952-2957.

8. Friel-Patti S, DesBarres K, Thibodeau L. Case studies using Fast ForWord. Am J Speech Lang Pathol 2001; 10: 203215.

9. Heiervang E, Stevenson J, Hugdahl K. Auditory processing in children with dyslexia. J Child Psychol Psychiatry 2002; 43: 931-938.

10. Share D, Jorm AF, MacLean R, Matthews R. Temporal processing and reading disabilities. Reading Writing: Interdisciplinary J 2003; 15: 151-178.

11. Agnew JA, Dorn C, Eden GF. Effect of intensive training on auditory processing and reading skills. Brain Lang 2004; 88: 21-25.

12. Tallal $P$. Improving language and literacy is a matter of time. Nat Rev Neurosci 2004; 5: 721-728.

13. McAnally KI, Castles A, Bannister S. Auditory temporal pattern discrimination and reading ability. $J$ Speech Lang Hear Res 2004; 47: 1237-1243.

14. Banai K, Ahissar M. Auditory processing deficits in dyslexia: task or stimulus related? Cereb Cortex 2006; 16: 17181728.

15. Tallal P, Piercy M. Developmental aphasia: impaired rate of non-verbal processing as a function of sensory modality.
Neuropsychologia 1973; 11: 389-398.

16. Neter J, Kutner MH, Nachtsheim CJ, Li W. Applied linear statistical models. 5th edn. Chicago: Irwin; 2005.

17. Tallal P, Miller S, Fitch RH. Neurobiological basis of speech: a case for the preeminence of temporal processing. Ann $N$ Y Acad Sci 1993; 682: 27-47.

18. Nagarajan S, Mahncke H, Salz T, Tallal P, Roberts T, Merzenich MM. Cortical auditory signal processing in poor readers. Proc Natl Acad Sci U S A 1999; 96: 6483-6488.

19. Ben-Yehudah G, Banai K, Ahissar M. Patterns of deficit in auditory temporal processing among dyslexic adults. Neuroreport 2004; 15: 627-631.

20. Heim S, Freeman RB Jr, Eulitz C, Elbert T. Auditory temporal processing deficit in dyslexia is associated with enhanced sensitivity in the visual modality. Neuroreport 2001; 12: 507510.

21. Simon C, Fourcin AJ. A cross-language study of speechpattern learning. J Acoust Soc Am 1978; 63: 925-935.

22. Chermak GD, Musiek FE. Central auditory processing disorders: new perspectives. San Diego: Singular Publishing Group San Diego Inc.; 1997.

23. Berwanger D, Wittmann M, von Steinbuchel N, von Suchodoletz W. Measurement of temporal-order judgment in children. Acta Neurobiol Exp 2004; 64: 387-394.

24. Frasca MFSS. Processamento auditivo em teste e reteste: confiabilidade da avaliação. [Master's thesis]. São Paulo: Faculdade de Medicina, USP; 2005. 